\title{
Development of Cubic Bezier Curve and Curve-Plane Intersection Method for Parametric Submarine Hull Form Design to Optimize Hull Resistance Using CFD
}

\author{
Deddy Chrismianto $^{1 *}$, Ahmad Fauzan Zakki ${ }^{1}$, Berlian Arswendo ${ }^{1}$ and Dong Joon Kim ${ }^{2}$ \\ 1. Department of Naval Architecture, Diponegoro University, Semarang 50275, Indonesia \\ 2. Department of Naval Architecture and Systems Marine Engineering, Pukyong National University, Busan 48513, South Korea
}

\begin{abstract}
Optimization analysis and computational fluid dynamics (CFDs) have been applied simultaneously, in which a parametric model plays an important role in finding the optimal solution. However, it is difficult to create a parametric model for a complex shape with irregular curves, such as a submarine hull form. In this study, the cubic Bezier curve and curve-plane intersection method are used to generate a solid model of a parametric submarine hull form taking three input parameters into account: nose radius, tail radius, and length-height hull ratio $(L / H)$. Application program interface (API) scripting is also used to write code in the ANSYS DesignModeler. The results show that the submarine shape can be generated with some variation of the input parameters. An example is given that shows how the proposed method can be applied successfully to a hull resistance optimization case. The parametric design of the middle submarine type was chosen to be modified. First, the original submarine model was analyzed, in advance, using CFD. Then, using the response surface graph, some candidate optimal designs with a minimum hull resistance coefficient were obtained. Further, the optimization method in goal-driven optimization (GDO) was implemented to find the submarine hull form with the minimum hull resistance coefficient $\left(C_{t}\right)$. The minimum $C_{t}$ was obtained. The calculated difference in $C_{t}$ values between the initial submarine and the optimum submarine is around $0.26 \%$, with the $C_{t}$ of the initial submarine and the optimum submarine being 0.00150826 and 0.00150429 , respectively. The results show that the optimum submarine hull form shows a higher nose radius $\left(r_{n}\right)$ and higher $L / H$ than those of the initial submarine shape, while the radius of the tail $\left(r_{t}\right)$ is smaller than that of the initial shape.

Keywords: submarine hull form; parametric design; cubic Bezier curve; curve-plane intersection method; hull resistance coefficeint; parametric design; goal-driven optimization (GDO); computational fluid dynamic (CFD); ANSYS
\end{abstract}

Article ID: 1671-9433(2015)04-0000-00

\section{Introduction}

Recently, the relationship between parametric design and

\footnotetext{
Received date: $2014-12-10$

Accepted date: 2015-04-08.

Foundation item: Supported by the Ministry of Research, Technology, and Higher Education Republic of Indonesia, through the Budget Implementation List (DIPA) of Diponegoro University, Grant No. DIPA-023.04.02.189185/2014, December 05, 2013.

*Corresponding author Email: deddychrismianto@yahoo.co.id

(c) Harbin Engineering University and Springer-Verlag Berlin Heidelberg 2015
}

ship performance analysis using CFD has become a topic of interest, specifically, the method of obtaining an optimum ship hull form that will ensure good hydrodynamic performance of a ship. Several methods of parametric design have been used in ship modeling. The use of the control points of the cubic B-spline to generate the parameters of ship hull design has been introduced with successful results (Sarioz, 2006; Mancuso, 2006; Perez et al., 2007; Ping et al., 2008, Perez and Clemente, 2011). Campana et al. (2006) used the Bezier polynomial patches method, in which the shape modification was controlled by a given number of control points that were used as the design variables/input parameters for finding the optimum shape in the optimization process. Chen and Huang (2004) used a technique for parameter estimation using the B-spline surface fitting method in an inverse design problem of finding the optimal hull form. In addition, Kang and Lee (2010) implemented the parametric morphing technique to rapidly generate a hull form with some variations of the input parameters. Furthermore, Rodriguez and Jambrina (2012) developed a programmed design based on a programming language as a tool for parametric hull form generation. In general, these methods can be used to create the parametric design for surface modeling only. However, some CFD software (especially the RANSE solver) needs a solid modeling at the meshing stage before the CFD analysis stage can begin. Pecot et al. (2012) introduced the parametric design of ship hull shape for solid modeling in the optimization calculation using CFD. In this design, CAD is used to generate some bulbous bow shapes based on the parameters for each design of experiment (DOE). Blanchard et al. (2013) used the movement of control points to create bulbous bow shapes in solid modeling according to two parameters: bow length and bow thickness. However, neither of these authors considered how to automatically generate the hull or bulbous bow shape.

In this study, some previous studies concerning ship performance analysis using CFD, especially for the free surface case, are used as a reference for the calculation of ship resistance. Seo et al. (2010) investigated flexible meshing techniques (i.e., a hybrid meshing for complex 
geometry regions and a sliding mesh for the rotating propeller); the meshing was employed to perform the three primary tests for ship resistance and propulsion performance. Unstructured meshing was used for the bow and stern regions with structured meshing for the remaining region. Non-conformal interfaces were placed between the sub-domains with different cell types. Wood et al. (2007) presented the results of the computations performed in the ETSIN for different ships with the RANSE free-surface commercial solver CFX. Some of the computational results were validated against experimental data in terms of various global and local quantities; the turbulence model used in the calculations was the shear stress transport (SST) model, and the volume of fluid method was used to model the free-surface flow.

For the optimization analysis, Kim and Yang (2010) studied the development of an efficient and effective hull surface modification technique for CFD-based hull form optimization using two methods: the radial basis function interpolation and the sectional area curve of the hull. The hull surface modification technique developed in this study is used to vary the hull forms during the optimization process, during which the objective functions associated with the resistance are evaluated by a practical design-oriented CFD tool (SSF) and a multi-objective genetic algorithm. Diez et al. (2010) ; Grigoropoulos and Chalkias (2010) developed a multidisciplinary robust design optimization (MRDO) procedure. The aim was to analyze the combined effects of considering several disciplines and uncertainty in ship design problems. Here, an MRDO problem was defined and solved, using a probabilistic sailing scenario, in terms of cruise speed, heel and yaw angles. In this study, robust design optimization is conducted using goal driven optimization (GDO) in the ANSYS environment, by choosing a multi-objective genetic algorithm (MOGA) to obtain the optimum bulbous bow shape.

The CAD-integrated CFD and optimization method can be used simultaneously to solve many problems, including the optimization of hull shapes. Chrismianto (2013); Chrismianto and Kim (2014) developed the parametric bulbous bow design, constructed using the cubic Bezier curve and the curve-plane intersection methods. It can be used to automatically generate a parametric bulbous bow shape using solid modeling for the optimization calculation in an effort to minimize the ship's resistance. The main objective of this study is to develop a submarine optimization design to obtain the best performance of total resistance using the cubic Bezier curve method and the curve-plane intersection method. Both methods are created using the API script programming code as a user defined function (UDF) in ANSYS Workbench.

\section{Parameters in submarine design}

The size and shape of the hull of a submarine plays an important role in determining its hydrodynamic performance. The ratio of length $(L)$ to height $(H)$ is an important variable in determining the hydrodynamic performance, particularly in relation to the total resistance of the hull when submerged in water. Karim et al. (2008) investigated the significant influence of various length-height ratios $(L / H)$ of the submarine hull DREA on viscous drag. In which the resistance that is reduced at the nose area of submarine so the submarine can be said to have a not noisy criteria (very quiet) allowing it to move undetected by radar, while the shape of the tail affects the shape of a wake at the back of the submarine. To get the best hydrodynamic performance, in particular, minimum viscous drag and maximum nominal wake fraction, Suman et al. (2010) considered diameter of the nose $\left(d_{n}\right)$ and tail $\left(d_{t}\right)$ as model hull geometric parameters while finding the optimum design of autonomous underwater vehicles (AUVs). And Parsons et al. (1974) developed a method for designing minimum drag hull shapes of axisymmetric submarine bodies. Nose radius $\left(r_{n}\right)$, diameter of middle body $(D)$, and tail radius are considered and modified to generate axisymmetric bodies.

Referring to previous research by Karim et al. (2008) and Sunan et al. (2010), the variation of a submarine hull form can be defined by three input parameters to create a parametric submarine (Fig. 1). These are as follows:

1) $L / H$.

2) The radius of submarine nose $\left(r_{n}\right)$.

3) The radius of submarine tail $\left(r_{t}\right)$.

In $3 \mathrm{D}$ solid modeling software such as the ANSYS design modeler, a solid model is generated from planes that contain one or more curves. Thus, in this study, there are some constraints that need to be considered in advance: a solid model is built using the lofting command, and the parameters of submarine modification are driven by curves in the vertical and transverse planes of the submarine hull form.

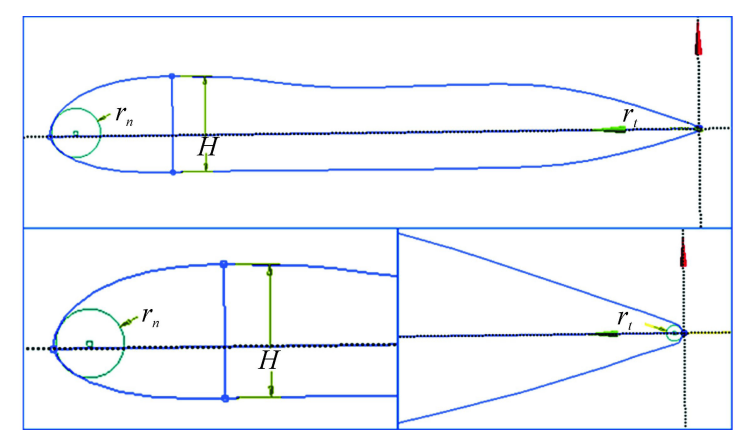

Fig. 1 Submarine hull form parameters

\section{Numerical overview}

The cubic Bezier curve is generally reliable for representing forms in most engineering applications. It has different properties such as tangents, normals, and curves, which are easily computed, and they ensure the continuity of a composite curve up to the second order (Choi, 1991; Lu and Huang, 1998; Piegl and Tiller, 1997; Saxena and Sahay, 
2005).

The Bernstein polynomials are applied to generate a Bezier curve. They are also referred to as Bezier basis functions and are used in defining a degree- $n$ Bezier curve by blending $n+1$ control vertices; they can be defined by

$$
B_{i}^{n}(u)=\frac{n !}{(n-i) ! i !} u^{i}(1-u)^{n-i}
$$

Based on the Bernstein polynomials at third degree, the cubic Bezier curve can be defined as follows:

$$
r(u)=B_{0}^{3}(u) V_{0}+B_{1}^{3}(u) V_{1}+B_{2}^{3}(u) V_{2}+B_{3}^{3}(u) V_{3}
$$

where

$$
\begin{aligned}
& B_{0}^{3}(u)=(1-u)^{3}, \quad B_{1}^{3}(u)=3 u(1-u)^{2} \\
& B_{2}^{3}(u)=3 u^{2}(1-u), \quad B_{3}^{3}(u)=u^{3}
\end{aligned}
$$

Here, the control points $\left(V_{0}, V_{1}, V_{2}\right.$, and $\left.V_{3}\right)$ can be explained as follows:

$V_{0}$ : start point of the curve segment, $P_{0}$.

$V_{1}$ : one-third point on starting tangent vector, $\left(V_{0}+t_{0 / 3}\right)$.

$V_{2}$ : two-third point on ending tangent vector, $\left(V_{3}-t_{1 / 3}\right)$.

$V_{3}$ : end point of the curve segment, $P_{1}$.

These definitions can be also written as follows:

$$
\begin{gathered}
V_{0}=P_{0}, \quad V_{1}=\left(V_{0}+t_{0} / 3\right) \\
V_{2}=\left(V_{3}-t_{1} / 3\right) P_{0}, \quad V_{3}=P_{1}
\end{gathered}
$$

Thus, it can be seen that there is the following relationship between the end points and end tangents $\left(P_{0}, P_{1}\right.$, $t_{0}$, and $\left.t_{1}\right)$ to the control points $\left(V_{0}, V_{1}, V_{2}\right.$, and $\left.V_{3}\right)$ :

$$
\begin{array}{cl}
P_{0}=V_{0}, & P_{1}=V_{3} \\
t_{0}=3\left(V_{1}-V_{0}\right), & t_{1}=3\left(V_{3}-V_{2}\right)
\end{array}
$$

In solid 3D software, a solid model is created from 2D curves. These curves must be sketched in some planes (vertical or transverse) before a solid body is generated using the loft, extrude, or sweep method. Here, the curve-plane intersection method is implemented to determine a common point that can be captured in two or more different planes. The general form of the equation of a plane is expressed as follows:

$$
a x+b y+c z-d=0 \quad \text { or } \quad \boldsymbol{r} \cdot \boldsymbol{p}=d \quad|\boldsymbol{p}|=1
$$

where $\boldsymbol{r}=(x, y, z)$ : coordinate variables, $\boldsymbol{p}=(a, b, c)$ : unit normal vector of the plane $\pi$, and $a^{2}+b^{2}+c^{2}=1$.

Thus, the intersection between a plane and a parametric curve is given by

$$
0=\boldsymbol{r}(t) \cdot \boldsymbol{p}-d, \quad 0=a x(t)+b y(t)+c z(t)-d
$$

and the procedure of curve-plane intersection can be explained as follows:

1) Input: $t_{0}$ (initial point), $p$ (unit normal of $\pi$ ), $d$ (distance of $\pi$ ).

2) Determine an estimated intersection point $\hat{t}$.
3) If $|\boldsymbol{r}(\hat{t}) \cdot \boldsymbol{p}-d| \leq \varepsilon$, return $\hat{t}$.

4) Else, $t_{0}=\hat{t}$ and go to 2).

The initial estimation point $t_{0}$ can be found using the Lattice evaluation method, which is as follows:

If,

$$
\left[\boldsymbol{p} \cdot \boldsymbol{r}\left(t_{i}\right)-d\right] \cdot\left[\boldsymbol{p} \cdot \boldsymbol{r}\left(t_{i+1}\right)-d\right]<0
$$

then $t_{i}$ or $t_{i+1}$ becomes the initial estimation point.

However, the estimated intersection point $\hat{t}$ is obtained as follows:

$$
\hat{t}=t_{0}-\left(\boldsymbol{p} \cdot \boldsymbol{r}\left(t_{0}\right)-d\right) /(\dot{\boldsymbol{r}} \cdot \boldsymbol{p})
$$

where

$$
\dot{\boldsymbol{r}}=\left.\frac{\mathrm{d} \boldsymbol{r}(t)}{\mathrm{d} t}\right|_{t=t_{0}}
$$

In addition, $\boldsymbol{p}$ and $d$ are coefficients of plane equation.

\section{Application of cubic Bezier curve and curve-plane intersection methods to parametric submarine design}

The parametric design of a solid model for submarines is proposed on the basis of four successive steps as follows:

1) Capturing curves from the existing submarine model.

2) Duplicating curves using the cubic Bezier curve.

3) Finding the intersection of the curve-plane.

4) Automatic parametric generation of the submarine model.

4.1 Capturing curves from the existing submarine model

From the existing submarine model, half the model can be cut and the other half can be hidden while the curves of the submarine shape are captured. To create these curves, two types of planes must be taken into account: vertical and transverse. In each plane, a 2D curve was captured. In this case (as shown in Fig. 2), the planes were divided and captured to include one curve in the vertical plane and 12 curves in the transverse plane.

The modeling procedure described in Fig. 2 was conducted using the ANSYS design modeler. A solid model of the submarine was modified and manipulated to obtain a parametric submarine model. It is applied in the CFD optimization calculation for the minimum total resistance.

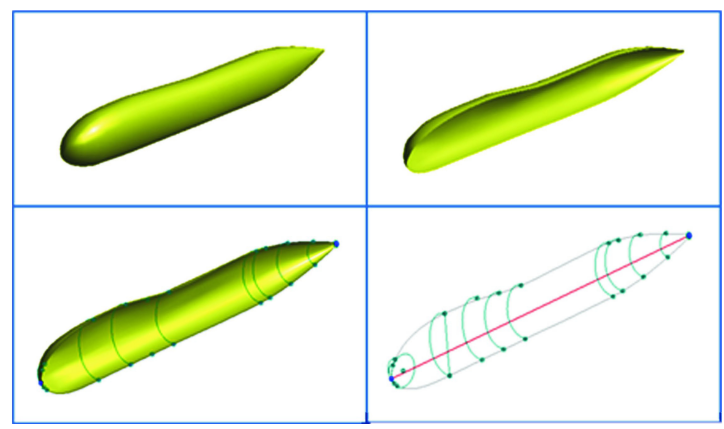

Fig. 2 Capturing curves from the solid submarine model 


\subsection{Duplicating curves using the cubic Bezier curve}

The cubic Bezier curve was applied to duplicate the existing curves. These curves were developed using API script to obtain identical curves with the same tangentials in both directions. A magnitude vector was fitted at the meeting point of the two curves. The procedure of duplicating curves can be explained as follows:

1) Determine the number of curves that will be created using the cubic Bezier curve.

2) Determine the location of internal control points $\left(V_{1}, V_{2}\right)$ in each curve referring to a given end tangent.

3) Build a new curve using API scripting in the ANSYS design modeler and compare the new and existing curves.

\subsubsection{Determining the number of curves that will be created using the cubic Bezier curve}

Usually, a parametric model is generated and controlled automatically by defined parameters. In this study, the defined parameters of a submarine hull form are created using the cubic Bezier curve method. This method was used to obtain a smooth shape in the solid model for a requirement of CFD and optimization analysis in the ANSYS workbench environment.

In this case, three parameters of the submarine design were implemented: $L / H, r_{n}$, and $r_{t}$. To realize this design, as shown in Fig. 3, there were 10 curves in the vertical plane view, separated by six dynamic points. $A_{1}$ and $A_{2}$ are the dynamic points that denote the radius of the submarine nose. $A_{3}$ and $A_{4}$ are the dynamic points that denote the maximum height of the submarine. $A_{9}$ and $A_{10}$ are the dynamic points that denote the radius of the submarine tail. All of them move up or down in the vertical plane. This affects the overall convexity of the submarine. Furthermore, to build a submarine model using the lofting method in the ANSYS design modeler, some transverse planes were required. These planes contain one curve or one point in each plane.

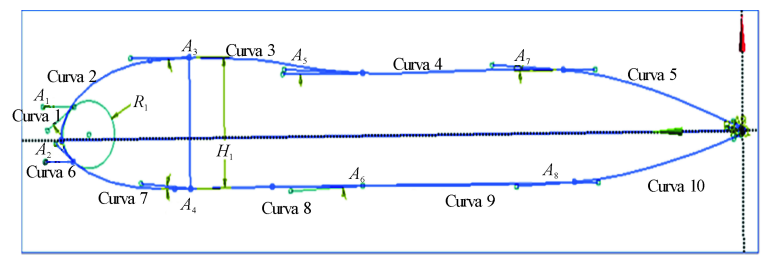

Fig. 3 Determining the number of curves, points, and angle of the end tangent for end points at every curve in the vertical plane

\subsubsection{Determining the location of internal control points $\left(V_{1}\right.$,} $\left.V_{2}\right)$ in each curve referring to a given end tangential

The cubic Bezier curve uses the four control points $\left(V_{0}\right.$, $V_{1}, V_{2}$, and $V_{3}$ ) to draw a curve; the two endpoints that are identified have been referred to as $V_{0}$ and $V_{3}$. The location of the internal control points $\left(V_{1}\right.$, and $\left.V_{2}\right)$ can be approximated on the basis of the end tangent value in which every end tangent has a magnitude and direction. The relationship of the internal control points $\left(V_{1}, V_{2}\right)$ and the end tangent $\left(t_{0}\right.$ and $t_{1}$ ) shown in Eq. (4b) can be written as follows:

$$
V_{1}=t_{0} / 3+V_{0}, \quad V_{2}=V_{3}-t_{2} / 3
$$

To assign a magnitude and a direction to each end tangent, $V_{1}$ and $V_{2}$ are applied in the $x$-axis and $y$-axis, respectively; therefore, Eq. (8) can be derived as shown below:

1) $V_{1}$ in the $x$-axis and $y$-axis:

$$
V_{1 x}=\frac{L_{1} \cos A_{1}}{3}+V_{0}, \quad V_{1 y}=\frac{L_{1} \sin A_{1}}{3}+V_{0}
$$

2) $V_{2}$ in the $x$-axis and $y$-axis:

$$
V_{2 x}=V_{3}-\frac{L_{2} \cos A_{2}}{3}, \quad V_{2 y}=V_{3}-\frac{L_{2} \sin A_{2}}{3}
$$

where $L_{1}$ and $L_{2}$ represent the magnitude of end tangents $\left(t_{0}\right.$, and $t_{1}$ ), and $A_{1}$ and $A_{2}$ represent the angles of end tangents ( $t_{0}$ and $t_{1}$ ).

Furthermore, all the existing curves (in the vertical and transverse planes) can be estimated for each location of the internal control points $\left(V_{1}, V_{2}\right)$ by analyzing the angle found using the dimensional command in the ANSYS design modeler. To maintain the continuity condition on two connected curves, their relationship has to satisfy the prerequisite of continuity. In other words, it can be said that the control point $V_{3}$ in curve 1 is equal to the control point $V_{0}$ in curve 2 , such that the tangent $t_{1}$ in curve 1 is equal to the tangent $t_{0}$ in curve 2 and so forth for the other curves.

\subsubsection{Building a new curve using API script and comparing the new and existing curves}

Once all the control points of each curve in each plane have been defined, new curves can be generated by duplicating all the control points of the existing curves using API. Here, all new curves were developed by applying the cubic Bezier curve, as defined in Eq. (2).

The result of the above-mentioned algorithm demonstrates that each curve in the vertical plane has been duplicated properly and smoothly. To achieve a reliable duplication of the new curve from the existing curve, the magnitude of the end tangent vector $\left(L_{1}, L_{2}\right)$ plays an important role in defining the location of the internal control points $\left(V_{1}\right.$ and $\left.V_{2}\right)$. If the magnitude is chosen appropriately, it will produce a reliable duplication of the new curve, although small deviation still exists. This is shown in Fig. 4, in which the new curves (designated by points) have curve forms that are very close to the existing curves.

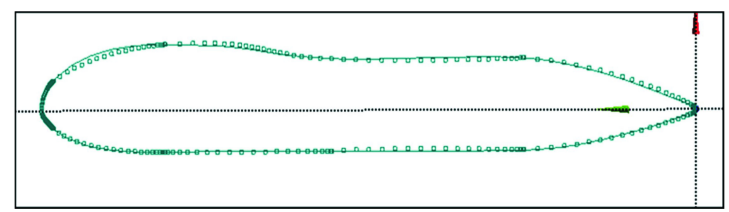

Fig. 4 Comparing new curves and existing curves (new curves as points; existing curves as lines)

\subsection{Finding the intersection of the curve-plane}

A surface or solid modeling of a submarine can be generated from curves using the lofting method in the ANSYS design modeler software. To generate this solid model, curve-plane intersection (in Eqs. (5b)-(7b)) was 
implemented to determine the intersection points, which were then captured by every transverse plane.

To obtain a curve with the same shape as the existing curve, all the end tangent vectors (both magnitude and direction) that have been defined in the duplicating curves section must be applied again to determine the internal control points $\left(V_{1}\right.$ and $\left.V_{2}\right)$. Finally, the cubic Bezier curve can be generated.

\subsection{Automatic parametric generation of the submarine model}

This procedure is essential along with those mentioned above. In this step, the parameters of the submarine solid model were generated on the basis of three input parameters. It was expected that the submarine shape would vary following a change in one or more input parameters. Changes to the submarine model should maintain the smoothness of the submarine shape.

The sequence of the automation stage of the submarine parametric design was initiated from the ratio of the length to maximum height, nose radius, and tail radius. In this case, a solid submarine model was constructed from transverse planes using the lofting command. It was difficult to solve this problem without simplifying the building of the solid model when some input parameters were changed. For simplicity, in the automation of the end tangent vector, the magnitude and direction at all the dynamic points that could be moved were assumed to be the same as those at the initial submarine curves.

Fig. 5 shows the result of the parametric submarine design in solid modeling. This result has been automatically generated using three input parameters.

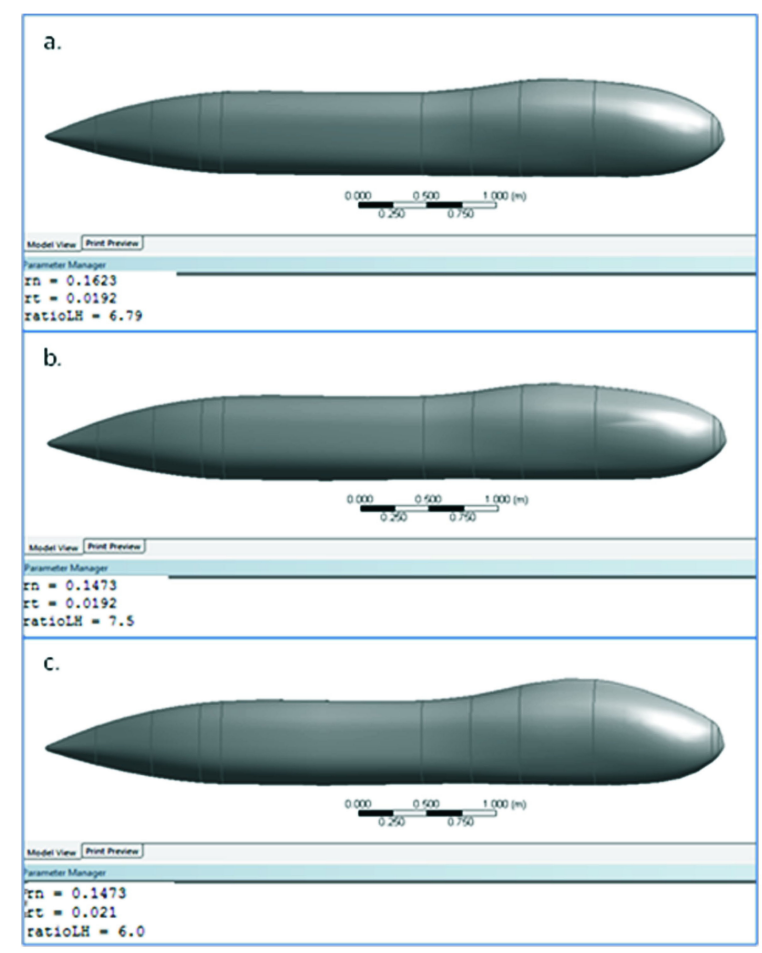

Fig. 5 Example of parametric submarine design using three variations of input parameters

\section{Example of submarine design optimization to minimize total resistance}

An example of the application is presented here to show that the proposed method can be applied easily and efficiently to a submarine. The purpose of this example is to determine the optimum submarine hull form with a minimum total resistance.

The optimization calculation can be performed using GDO in the ANSYS environment, where MOGA is chosen to give the optimum submarine shape. Five sequential steps must be completed for optimization analysis: defining input parameters and output parameters, defining the design space for each of the input parameters (such as the minimum and maximum values), creating DOE, creating a response surface for each output parameter, and applying optimization calculations and setting the objective function.

Input parameters include $L / H, r_{n}$, and $r_{t}$. The coefficient of total resistance $\left(C_{t}\right)$ is defined as the output parameter. The design space of the input parameters was defined at about $\pm 10 \%$, as shown in Table 1 . The number of DOE was defined by referring to the number of input parameters $(N)$. If there are three input parameters, the DOE number is equal to 14 design points (Table 2).

The response surfaces show the variation of each output parameter with respect to input parameters at a given time (Fig. 6).

Fig. 6 shows the response surfaces that represent the relationship between the coefficient of total resistance as an output parameter with all the input parameters for each DOE type. The response surface graph shows some candidate optimal designs with a minimum $C_{t}$ value marked in blue. Further, the optimization calculation must be analyzed to obtain the optimal design from some candidates.

Finally, the minimum $C_{t}$ value was obtained. The calculated difference in $C_{t}$ values between the initial submarine and optimum submarine is around $0.26 \%$, with the $C_{t}$ of the initial submarine and the optimum submarine being 0.00150826 and 0.00150429 , respectively. This result can be also explained by the comparison of forces around the submarine (Fig. 7). This shows that the force around the hull of the optimum submarine is smaller than that around the initial one (the red area around the submarine hull form on the optimum submarine decreases).

The comparison of the parameters of the optimum shape and the initial shape is shown in Fig. 8. Fig. 8 shows that the optimum submarine hull form has a higher $r_{n}$ and higher $L / H$ than those of the initial submarine shape, while $r_{t}$ is smaller than that of the initial shape. 
Table 1 The design space of input parameters

\begin{tabular}{|c|c|c|c|c|}
\hline \multicolumn{2}{|c|}{ Parameter } & Initial & Minimun & Maximum \\
\hline \multicolumn{2}{|c|}{ Nose ratio $r_{n}$} & 0.1623 & 0.14607 & 0.17853 \\
\hline \multicolumn{2}{|c|}{ Tail ratio $r_{t}$} & 0.0192 & 0.01728 & 0.02112 \\
\hline \multicolumn{2}{|c|}{ Ratio $L / H$} & 6.790 & 6.111 & 7.469 \\
\hline \multicolumn{5}{|c|}{ Table 2 Calculating the design of experiment (DOE) } \\
\hline No. & $\begin{array}{c}\text { Nose ratio } \\
r_{n}\end{array}$ & $\begin{array}{c}\text { Tail ratio } \\
r_{t}\end{array}$ & Ratio $L / H$ & $C_{t}$ \\
\hline 1 & 0.162300 & 0.019200 & 6.79000 & 0.00150826 \\
\hline 2 & 0.146070 & 0.019200 & 6.79000 & 0.00151598 \\
\hline 3 & 0.178530 & 0.019200 & 6.79000 & 0.00150835 \\
\hline 4 & 0.162300 & 0.017280 & 6.79000 & 0.00150738 \\
\hline 5 & 0.162300 & 0.021120 & 6.79000 & 0.00150741 \\
\hline 6 & 0.162300 & 0.019200 & 6.11100 & 0.00152246 \\
\hline 7 & 0.162300 & 0.019200 & 7.46900 & 0.00150451 \\
\hline 8 & 0.149104 & 0.017639 & 6.23795 & 0.00152504 \\
\hline 9 & 0.175496 & 0.017639 & 6.23795 & 0.00151337 \\
\hline 10 & 0.149104 & 0.020761 & 6.23795 & 0.00152634 \\
\hline 11 & 0.175496 & 0.020761 & 6.23795 & 0.00151353 \\
\hline 12 & 0.149104 & 0.017639 & 7.34205 & 0.00150969 \\
\hline 13 & 0.175496 & 0.017639 & 7.34205 & 0.00150701 \\
\hline 14 & 0.149104 & 0.020761 & 7.34205 & 0.00150899 \\
\hline 15 & 0.175496 & 0.020761 & 7.34205 & 0.00150598 \\
\hline
\end{tabular}
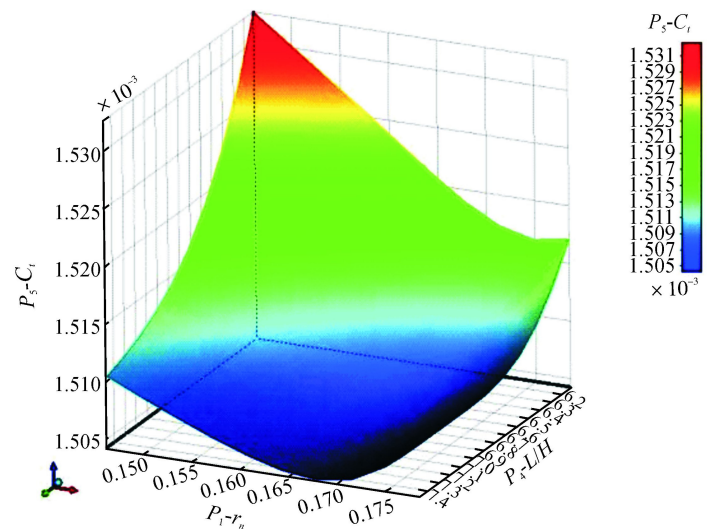

(a) Response chart 1

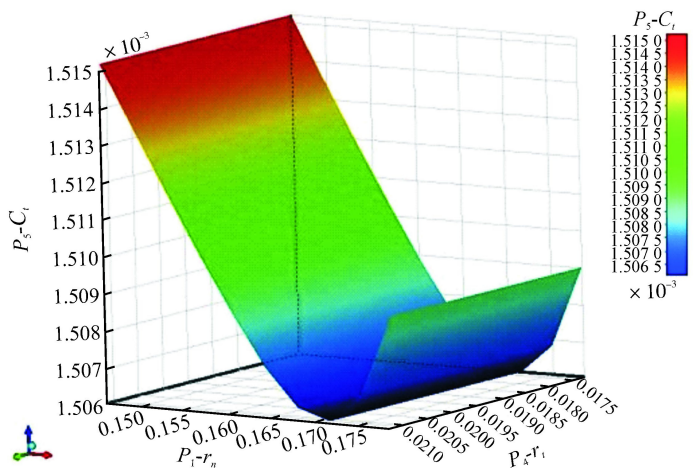

(b) Response chart 2

Fig. 6 Response surface between input parameters and output parameter

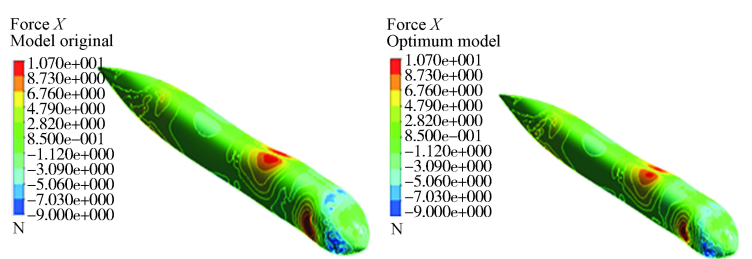

Fig. 7 Force around the hull: initial (a) and optimum (b)

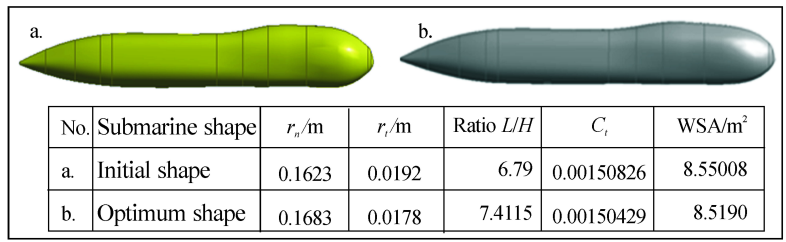

Fig. 8 The comparison of parameters of the submarine shapes

\section{Conclusions}

In this study, a parametric submarine design was performed with three input parameters: $L / H, r_{n}$, and $r_{t}$. In addition, four successive steps must be followed to obtain the desired result: capturing curves from an existing submarine model, duplicating the curves using the cubic Bezier curve, finding the curve-plane intersection, and creating an automatic parametric generation of the submarine. Particularly, after duplicating the curves using the cubic Bezier curves, all the end tangents of the curves (both magnitude and direction) must be defined correctly following the initial submarine shape to maintain the requirement of continuous condition $\mathrm{C} 1$. It was expected that the submarine obtained would have a smooth shape, similar to the initial submarine.

An example of the application of the ship resistance minimization using CFD has been presented to show that the proposed method can be successfully applied. A submarine hull form model was chosen for modification. Before the optimization step was processed, this model was analyzed in advance using CFD. Following this, the optimization method using GDO in the ANSYS environment was implemented to determine the optimum submarine hull form. The results show that the optimum submarine hull form has a higher $r_{n}$ and higher $L / H$ ratio than those of the initial submarine shape, while $r_{t}$ is smaller than that of the initial shape.

\section{References}

Blanchard L, Berrini E, Duvigneau R, Roux Y, Mourrain B, Jean E (2013). Bulbous bow shape optimization. V International Conference on Computational Methods in Marine Engineering (MARINE 2013), Hamburg, Germany, 1-12.

Campana EF, Peri D, Tahara Y, Stern F (2006). Shape optimization in ship hydrodynamics using computational fluid dynamics. Computer Methods in Applied Mechanics and Engineering, 196(1), 634-651.

DOI: $10.1016 /$ j.cma.2006.06.003 
Chen PF, Huang CH (2004). An inverse hull design approach in minimizing the ship wave. Ocean Engineering, 31(13), 1683-1712.

DOI: 10.1016/j.oceaneng.2003.08.010

Choi BK (1991). Surface modeling for CAD/CAM. Elsevier, Seoul, Korea.

Chrismianto D (2013). Parametric bulbous bow design for the minimization of ship resistance by using CFD. $\mathrm{PhD}$ thesis, Pukyong National University, Busan, Korea, 7-10.

Chrismianto D, Kim DJ (2014). Parametric bulbous bow design using the cubic Bezier curve and curve-plane intersection method for the minimization of ship resistance in CFD. Journal of Marine Science and Technology, 19(4), 479-492. DOI: 10.1007 s00773-014-0278-x

Diez M, Peri D, Fasano G, Campana EF (2010). Multidisciplinary robust optimization for ship design. $28^{\text {th }}$ Symposium on Naval Hydrodynamic, Pasadena, USA.

Grigoropoulos GJ, Chalkias DS (2010). Hull-form optimization in calm and rough water. Computer-Aided Design, 42(11), 977-984. DOI 10.1016/j.cad.2009.11.004

Kang JY, Lee BS (2010). Mesh-based morphing method for rapid hull form generation. Computer-Aided Design, 42(11), 970-976. DOI: $10.1016 /$ j.cad.2009.11.004

Karim MM, Rahman MM, Alim MA (2008). Numerical computation of viscous drag for axisymetric underwater vehicles. Jurnal Mekanikal, 26, 9-21.

Kim H, Yang C (2010). A new surface modification approach for CFD-based hull form optimization. International Conference on Hydrodynamics, Shanghai, China.

Lu WC, Huang JM (1998). Modification of a NURBS curve with nose features. Computer Integrated Manufacturing Systems, 11(4), 253-265.

DOI: $10.1016 / \mathrm{S} 0951-5240(98) 00023-8$

Mancuso A (2006). Parametric design of sailing hull shapes. Ocean Engineering, 33(2), 234-246.

DOI: $10.1016 /$ j.oceaneng.2005.03.007

Parsons JS, Goodson RE, Goldschmied FR (1974). Shaping of axisymmetric bodies for minimum drag in incompressible flow. Journal of Hydronautics, 8(3), 100-107.

DOI: $10.2514 / 3.48131$
Pecot F, Yvin C, Buiatti R, Maisonneuve JJ (2012). Shape optimization of a monohull fishing vessel. $12^{\text {th }}$ International Conference on Computer and IT Application in the Maritime Industries, Liege, Belgium, 7-18.

Perez F, Clemente JA (2011). Constrained design of simple ship hulls with B-spline surfaces. Computer-Aided Design, 43(12), 1829-1840. DOI: 10.1016/j.cad.2011.07.008

Perez F, Suarez JA, Clemente JA, Souto A (2007). Geometric modelling of bulbous bows with the use of non-uniform rational B-spline surfaces. Journal of Marine Science and Technology, 12(2), 83-94.

DOI: $10.1007 / \mathrm{s} 00773-006-0225-6$

Piegl L, Tiller W (1997). The NURBS book. Springer, Berlin, Germany.

Ping Z, Xiang ZD, Hao LW (2008). Parametric approach to design of hull forms. Journal of Hydrodynamics, 20(6), 804-810. DOI: 10.1016/S1001-6058(09)60019-6

Rodriguez A, Jambrina LF (2012). Programmed design of ship forms. Computer-Aided Design, 44(7), 687-696. DOI: 10.1016/j.cad.2012.03.003

Sarioz E (2006). An optimization approach for fairing of ship hull forms. Ocean Engineering, 33(16), 2105-2118. DOI: $10.1016 /$ j.oceaneng.2005.11.014

Saxena A, Sahay B (2005). Computer aided engineering design. Anamaya Publisher, New Delhi, India.

Seo JW, Seol DM, Lee JH, Rhee SH (2010). Flexible CFD meshing strategy for prediction of ship resistance and propulsion performance. International Journal of Naval Architecture and Ocean Engineering, 2(3), 139-145.

DOI: $10.3744 / J N A O E .2010 .2 .3 .139$

Suman KNS, Rao DN, Das HN, Kiran GB (2010). Hydrodynamic performance evaluation of an ellipsoidal nose for high speed under water vehicle. Jordan Journal of Mechanical and Industrial Engineering, 4(5), 641-652.

Wood MP, Gonzalez LM, Izquierdo J, Sarasquete A, Rojas LP (2007). RANSE with free surface computations around fixed DTMB 5415 model and other Baliño's fishing vessels. The 9th International Conference on Numerical Ship Hydrodynamics, Ann Arbor, Michigan, USA, 1-13. 